\author{
Janusz SZPYTKO ${ }^{1 *}$ \\ Pawel HYLA ${ }^{1}$ \\ Yorlandys SALGADO DUARTE ${ }^{1}$
}

\title{
AUTONOMOUS VEHICLES ENERGY BASED OPERATION CAPACITY PLANNING
}

\begin{abstract}
The paper presents an optimization model for an Automatic Guided Vehicle (AGV) operation capacity planning with focused to complete predicted mission. To successfully complete the mission the available resources related to the mission task we need to predict set of the device operation capacity indicator: technical status of the device structure and functions, device control strategy, access to the energetic resources type and others. Paper is focusing on device control strategy of the AGV under operation optimisation results minimizing possible gaps corresponded with the access to the energy. The scenarios are proposed by a Particle Swarm Optimization (PSO) algorithm, and the AGV operation is evaluated with the State of Charge (SoC) variable. The selected SoC variable allows us to describe the simulated operation in detail over time. The model output is the optimal trajectory for the AGV system considering the working environment and the satisfaction of the mission preestablished by the user. The inputs parameters of the optimization model are validated by a real environment created in a laboratory scale. The localization system, trajectories planning, workspace mapping and AGV control system concepts are briefly described, as well as the artificial intelligence used as methods and tools for AGV working control, to guide the discussion towards the contribution proposed.
\end{abstract}

\section{INTRODUCTION}

Transport, in its broadest sense, has always been a fundamental pillar for the development of civilization [1]. Todays, within the transport devices, AGVs and their applications in industry with hazard environment, draw the attention of many researchers. In AGVs, the ability to self-locate the transport device, i.e. its current location in the workspace, is the main way to configure the reliability and safety of these devices when working in real time [2].

Automatic routing problems $[2,3]$ require defining the boundary conditions that describe the workspace [4], connected to a coordinate system, i.e., the characteristics of the environment, selecting the optimal route path (routing) and the variety of possible tasks (scheduling) to be performed [5]. In addition, it is necessary to insert a certain range of interactivity, which allows the exchange of information between transport devices,

\footnotetext{
${ }^{1}$ AGH University of Science and Technology, Krakow, Poland

*E-mail: szpytko@agh.edu.pl

https://doi.org/10.36897/jme/130719
} 
the environment, and the human factor (Man-Machine Interface System) [6]. However, the most important problem in AGV routing is the control task. Each AGV control system and its algorithms (artificial intelligence depending on the technology) must be designed as a system that allows prevents critical situations.

The first fully automatic vehicles for industrial applications (formerly called driverless vehicles, DLV) were designed in 1954 [7]. The driverless electric car was produced by Barrett Electronics. The first industrial problems solved by DLV were moving loads in a warehouse in the state of South Carolina. The routing of the DLV vehicles was very simple. The path was mounted on a concrete floor with wires. The device followed the signal emitted by the wires secured in the concrete floor.

In the middle of the century, the first routing system was developed that replaced cables with lasers. In this system, reflective targets are mounted on the floor of the facility. In addition, the facility's workspace was provided by an $X, Y$ coordinate system. The laser navigation system works as follows: the basic coordinates of the workspace are loaded into the AGV's memory. A rotating mirror laser emits the laser beam. The laser light is used to measure the distance to another object in the workspace automatically. The data obtained is compared with the memory configuration (AGV position), resulting in the actual position of the vehicle. The AGV computer can then compare the calculated position with a previously planned route and correct the path if necessary.

Another new method of wireless navigation was introduced in the mid-1990s. This technology was called gyro navigation. In this solution, each AGV was equipped with a solid-state gyroscope. The actual location of the AGV was estimated based on deviations from the base route, stored in the AGV memory. In addition, magnetic grid markers with unique $X, Y$ coordinates were installed on the floor of the facility. These markers were used as reference points to correct any small errors accumulated in the travel distance from one point to another with the help of gyroscopic navigation.

Recent transport systems, enhanced with integrated solutions, especially telematics, contributed to the development of new routing methods and tools for AGVs. So-called electronic systems or e-Systems were developed for routing. E-Systems contributed to the development of a new type of automated control vehicle: UGV. UGVs are a complete family of vehicles that use modern tools with all the power of today's electronics to perform navigation tasks and intelligent decision-making processes. A conventional graph of the UGV routing system is shown in Fig. 1.

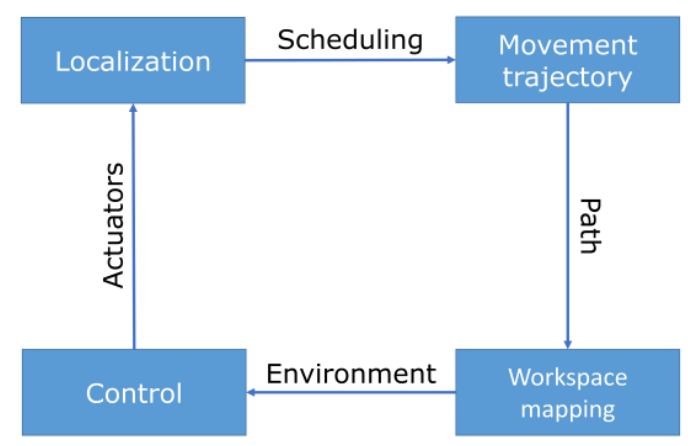

Fig. 1. UGV's navigation system mode adopted from [8] 
Today, the problem of configuring a routing system that works in real time mode for AGVs is the subject of research by many scientific units. The routing system is made up of four basic problems: Localization, Control, Workspace mapping and Movement trajectory as Fig. 1 shows. In this paper, decomposing the Lego problems in pieces, we focus the attention on movement trajectory problem, reason why, below, we briefly describe the main topics related with this field and the contribution proposed by us.

The main rule in AGV trajectory design is to plan the trajectory in advance. The route must lead the transport device from the start point (task start point) to the destination point (task end point) considering the device configuration, construction, and load dimension. In addition, the route must be planned with an optimization criterion based on the defined objective [9]. All optimization criteria in the path design process can be expressed by three basic criteria:

1. route criteria (minimizing the duration of the trip);

2. time criterion (minimize the time of task completion);

3. energy criterion (minimize the energy expenditure associated with the completion of the task).

Furthermore, it is possible to express additional criteria, through combinations of the above criteria. The essential problem of trajectory planning is to draw the geometric curve between the starting point and the destination. In addition, the curve must consider the constraints associated with the obstacles (movement cannot cause the collision) and must consider the structural constraints of the AGV. Within the group of AGVs it is possible to find unique vehicles with holonomic (see Fig. 2) and non-holonomic kinematic structure (see Fig. 3).

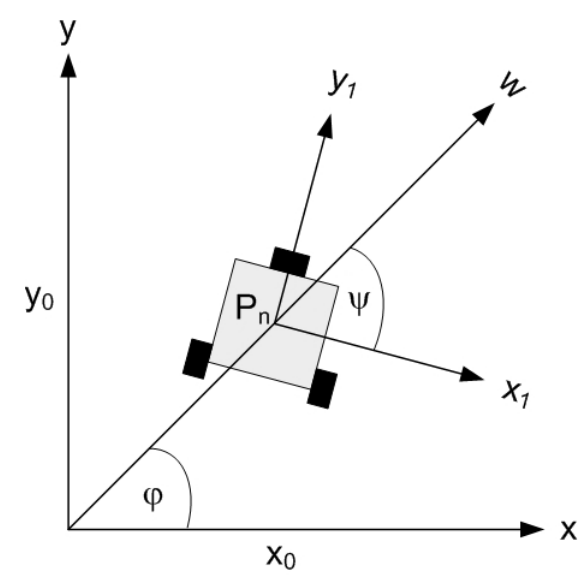

Fig. 2. AGV nonholonomic kinematic structure

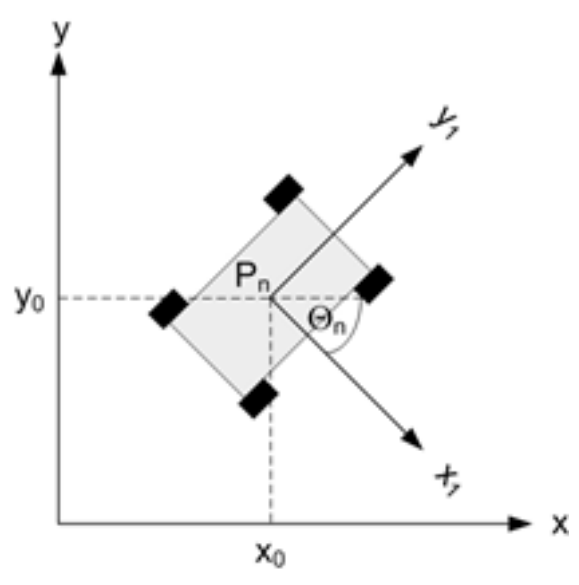

Fig. 3. AGV holonomic kinematic structure

The manoeuvrability of AGVs with non-holonomic kinematic structure can be compared to traditional car wheels construction. The second group of AGVs with holonomic kinematic structure are more flexibles. The independently driven wheels improve the manoeuvrability because they allow rotation around the axle itself and in some cases on the diagonal. Today, AGV structures with a holonomic kinematic structure are more common in real processes. However, regardless of the features of the $\mathrm{AGV}$, for the design of an 
autonomous vehicle system, the potential energy consumption and/or the recharge time to get back to work are the crucial parameters.

An AGV can also be called a laser-guided vehicle (LGV), depending on the technology. The lower cost versions of AGVs are often called automated guided carts (AGC) and are usually guided by magnetic tape. In this research, we use an AGV with these features that works in continuous operation (laboratory designed environment), and in the following sections we describe the scenario simulated in the laboratory and the parameters of the AGV. As we know, AGVs need batteries to operate autonomously. That is why, by measuring the battery level, it is possible to know by means of simulations, if AGVs can complete the planned mission. In this paper, we propose the SoC indicator to reflect the operating capacity of the $\mathrm{AGV}$, in our case, energy oriented, linking the planning strategies of the trajectory with energy access of the AGV to complete the mission, subject in our case to optimization.

The SoC indicator is not selected arbitrary and is a common subject of research when the relation between battery and AGV operation capacity is under discussion [10-13]. For instance, reference like [11] shows how through battery management of AGVs is possible increase the manufacturing capacities ( $\mathrm{SoC}$ is used as a state variable in the proposed model). The paper investigates how the duration of battery charging for AGVs can be varied to increase flexibility of a manufacturing system and the authors used a conventional acid battery in the analysis.

Other authors [12] with similar purposes propose new battery charging strategies and they introduce the lithium-ion battery in the discussion, and in the top of the iceberg a recent reference [13] describes a full example of the AGVs battery management application in a container terminal. As expected, when the problem to be solved is complex, there are assumptions and simplifications in place to find a solution in a reasonable time, and the reference [13] is one of the cases.

Taking into consideration the previous contributions and the reference [10] as a step earlier of the reference [13], we propose an in-depth analysis of a closer model to reality to assess the relation battery and operation capacity of the AGV, but additional remarks related to the battery charge are needed during the simulation process, and therefore to be considered in the optimization. AGVs use various battery charging options [10]. Each option depends on the user's preference:

1. battery swap: requires an operator to manually replace the discharged battery from the AGV with a fully charged battery in its place after approximately 8-12 hours (about one shift of work) of AGVs operation. 5-10 minutes is required to perform this task for one $\mathrm{AGV}$, as a standard time;

2. automatic and opportunity charging: this option allows continuous operation. On average, an AGV is charged for 12 minutes every hour for automatic charging and no manual intervention is required. If the opportunity is taken, the AGV will charged whenever the opportunity arises. When a battery pack reaches a predetermined level, the AGV will complete the current job assigned to it before going to the charging station;

3. automatic battery swap: this option is an alternative to manual battery swap. It requires an additional piece of automation machinery, an automatic battery changer, to the 
general AGV system. AGVs will approach the battery swap station and their batteries will be automatically replaced with fully charged batteries. The automatic battery changer then places the removed batteries into a charging slot for automatic recharging. The automatic battery changer tracks the batteries in the system and removes them only when they are fully charged.

While a battery swap system reduces the manpower required to swap batteries, recent developments in battery charging technology allow batteries to be charged more quickly and efficiently potentially eliminating the need to swap batteries.

In this work, we select the second option, automatic and opportunity charging, and through the SoC variable over time, we assess the AGV operation capacity status, simulating proposed scenarios and selecting the best.

The paper objective is to propose a model thought a sensitive indicator (SoC in our case) that allows to predict the planning of the operation capacity following an optimization criterion (energy is one of them). The criteria follow the three basic examples to design the movement trajectory of the AGV described above, but as the conclusion will describe and the body of the paper will reflect, energy-oriented indicators behave well in the selected scenario.

The following sections are organized as follows: firstly, the operation capacity model and the optimization problem formulation are presented with the constraints set. Then, by sections we parametrize and solve the scenarios proposed assessing the SoC variable over time (operation capacity indicator that describe technical status of the device) and describing the scenarios simulated with the corresponding set of initial conditions considered. Finally, some important conclusions are highlighted, specially the proposed SoC indicator proposed to evaluate the operation capacity of the AGV and the connection of the indicator with the planning trajectory process.

\section{MODEL DEFINITION}

It is necessary to evaluate the operating capacity of the AGV to know if the AGV can fulfil the assigned mission. We found the SoC indicator as an accepted measure to predict the operating capacity, energy oriented, to determine whether the AGV completes the mission. We selected the SoC indicator because autonomous AGV use batteries and this indicator describes the operating capacity status by the battery level of the AGV.

To simulate the AGV operation capacity, a generic high-voltage battery circuit implemented by MathWorks in MATLAB Simulink [14] is used and changed conveniently. The circuit allows us to estimate the SoC (\% percent), temperature in the battery (Celsius degrees), current (Ampere) and voltage (Volt) over the time depending on the work conditions. Figure 4 shows the block diagram implemented to simulate the AGV capacity operation. As Fig. 4 shows, the input of the block diagram (signal from the workspace of MATLAB) is changed to recreate the scenario proposed (manipulating the current flow defined by the optimization model), and the output of the block diagram (variable SoC exported to workspace of MATLAB) is used for the optimization model to assess the scenario (the SoC vector must be non-zero in all domain to complete the task successfully). 
In the case of the parameters of the circuit, all of them are changed to adapt the circuit to the parameters of the physical AGV in the laboratory.

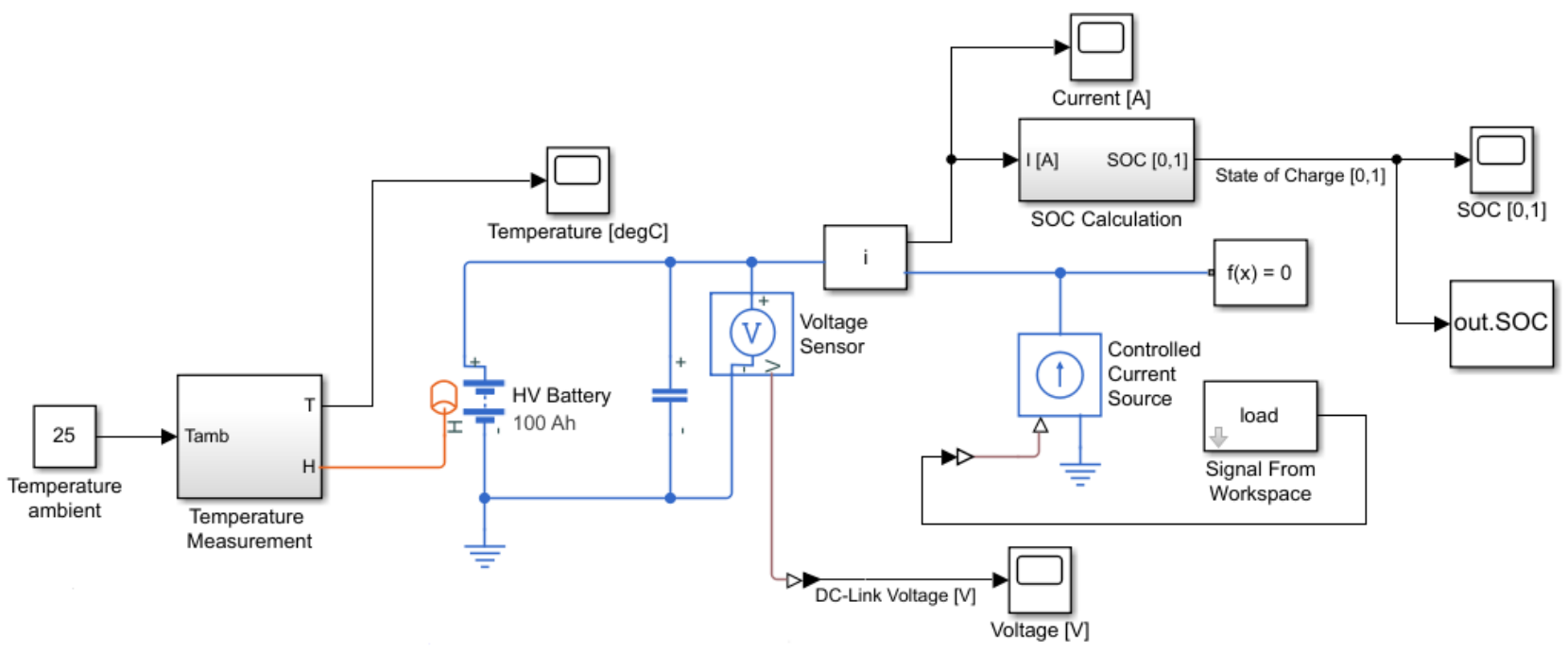

Fig. 4. Block diagram of the battery in the AGV system

The battery model used in the paper proposes the voltage according to the charge and has the following relationship:

where:

$$
V=V_{0}\left(\frac{S o C}{1-\beta(1-S o C)}\right)
$$

- SoC (State-of-Charge) is the ratio of current charge to rated battery capacity.

- $V_{0}$ is the voltage when the battery is fully charged at no load, as defined by the Nominal voltage parameter.

$-\beta$ is a constant that is calculated as follows: the battery voltage is $V_{1}$ when the charge is $A H_{1} . A H_{1}$ is the charge when the no-load (open circuit) voltage is $V_{1}$, and $V_{1}$ is less than the nominal voltage.

The Equation (1) defines an approximate relationship between voltage and remaining charge. This approximation replicates the increasing rate of voltage drop at low charge values and ensures that the battery voltage becomes zero when the charge level is zero. The advantage of this model is that it requires few parameters, which are readily available on most datasheets. The battery model used in the paper provide additional parameters to define battery behaviour at a second temperature. The extended equations for the voltage when the thermal port is exposed are:

$$
\begin{gathered}
V=V_{0 T}\left(\frac{S o C}{1-\beta(1-S o C)}\right) \\
V_{0 T}=V_{0}\left(1+\lambda_{V}\left(T-T_{1}\right)\right)
\end{gathered}
$$

where:

$-T$ is the battery temperature,

- $T_{1}$ is the nominal measurement temperature, 
$-\lambda_{V}$ is the parameter temperature dependence coefficient for $V_{0}$,

$-\beta$ is calculated in the same way before, using the temperature-modified nominal voltage $V_{0 T}$.

The internal series resistance, self-discharge resistance, and any charge-dynamic resistances are also functioning of temperature:

$$
R_{T}=R\left(1+\lambda_{R}\left(T-T_{1}\right)\right)
$$

where $\lambda_{R}$ is the parameter temperature dependence coefficient.

All the temperature dependence coefficients are determined from the corresponding values, means, nominal and second measurement temperatures. In addition, when the charge is dynamics (modelling perspective) in the model, the time constants vary with temperature in the same way. The battery temperature is determined from a summation of all the ohmic losses included in the model:

where:

$$
M_{t h} \dot{T}=\sum_{i} \frac{V_{T, i}^{2}}{R_{T, i}}
$$

- $\quad M_{t h}$ is the battery thermal mass,

- $\quad i$ corresponds to the $i$-th ohmic loss contributor. The losses include: series resistance, self-discharge resistance, first charge dynamics segment, second charge dynamics segment;

- $\quad V_{T, i}$ is the voltage drop across resistor $i$,

- $\quad R_{T, i}$ is resistor $i$.

All the additional parameters that are obtained as output of the circuit allows us to analyses in depth the changes in the variables of the circuit when the input is modified, in this case the current source that recreate the scenario. Once the diagram is described, it is necessary define the control of the current source that simulates the working condition of the AGV. Saying that, manipulating the current source, is possible simulate when the AGV is consuming current from the battery and when the battery is receiving power from an external source (operation process).

This control allows us to simulate several scenarios for the same AGV work regime and determine with an optimization model, what is the best scenario for this system, operation capacity perspective, given the route scheduling, the working conditions and model constraints (planning process).

We propose a simple optimization model to manipulate the current source. During the research, four different objective functions were tested: first one with objectives combined, minimizing the linear combination of the stop time tc and the number of charges $N t c$ in a work cycle $i$-th as shows Equation (6), the second one with objective combined, minimizing the linear combination of the stop time tc and the number of charges Ntc in a work cycle $i$-th, weighting the linear combination by the number of charges, as shows Equation (6a), and the third and fourth one with single objective, minimizing the area below of the SoC over the time and the weighting area below of the SoC over the time, respectively, as shows Equation (6b) and Equation (6c). For all the cases the constraints remain the same. 


$$
\begin{gathered}
\min F\left(t c_{i}\right)=\sum_{i=1}^{N} t c_{i} \times N t c_{i} \\
\min F\left(t c_{i}\right)=\eta \times \sum_{i=1}^{N} t c_{i} \times N t c_{i} \\
\min F(S o C)=\int_{t=0}^{t=T} S o C(t) d t \\
\min F(S o C)=\eta \times \int_{t=0}^{t=T} \operatorname{SoC}(t) d t
\end{gathered}
$$

Subject to $0 \leq t c_{i} \leq t c_{\text {max }_{-} i}$ and $S o C_{t}=1,2, \ldots, T>0$ (constraint to complete the task within a work cycle)

where:

- $t c$ is the stop time,

- Ntc number of charges, logical vector, equal to one when the charge is needed, zero overside,

$-i$ index of work cycle,

$-N$ number of work cycles,

$-t$ index of time,

- $T$ simulation window,

$-\eta=\sum_{i=1}^{N} N t c_{i}$ weighting parameter.

The objectives were chosen because by minimizing the combined criteria or the simple criteria, depending on the case, the system working time is affected as little as possible. Once the best scenario for this AGV system has been defined, it can be verified in the real system, if the best movement trajectory configuration proposed merge with the reality of the system.

The battery model is a based block diagram differential approximation and, as we know, the literature contains many battery models, therefore in future steps of the research testing other models will be a target. Like the battery model, challenging other criteria to optimize the scenario is within the future work, but this paper just tries to create the starting point of the investigation.

In this research, a Particle Swarm Optimization (PSO) algorithm based on group intelligence is proposed for the solution of the problem. The algorithm was proposed by Kennedy and Eberhart in 1995 for the solution of optimization problems [15]. Due to the simplicity of the PSO, it has been successfully applied to different problems that require the estimation of parameters in spaces of high dimensions. The growing number of applications in engineering systems motivated the selection of the PSO algorithm in this work. Other point in favour is the well-implemented version of the algorithm in MATLAB, the same tool used by the previously described operation capacity of the AGV. PSO algorithmic used in this investigation is based on the algorithm described in [15], using modifications suggested in [16].

PSO algorithm iterates until it reaches a stopping criterion, in this case, when the relative change in the best objective function value is less than $1.0000 \mathrm{e}-06$. The algorithm settings used in the solution of all the scenarios is described in the Table 1.

Once methods and models to be used are defined, in the following sections we describe the AGV available in the lab, the scenarios proposed and the solutions for all the objectives declared above. 
Table 1. Optimization algorithm properties

\begin{tabular}{|l|c|}
\hline \multicolumn{1}{|c|}{ Property } & Value \\
\hline Function Tolerance & $1.0000 \mathrm{e}-06$ \\
\hline Inertia Range & {$[0.1000,1.1000]$} \\
\hline Initial Swarm Span & 2000 \\
\hline Max Iterations & $200 \times($ number of variables $)$ \\
\hline Max Stall Iterations & 20 \\
\hline Min Neighbors Fraction & 0.2500 \\
\hline Self-Adjustment Weight & 1.4900 \\
\hline Social-Adjustment Weight & 1.4900 \\
\hline Swarm Size & Min $(100,10 \times$ number of variables $)$ \\
\hline
\end{tabular}

\section{MODEL PARAMETRIZATION}

Figures 5 shows the aerial overview of the AGV used in this investigation and Table 2 describes the general characteristics of the device.

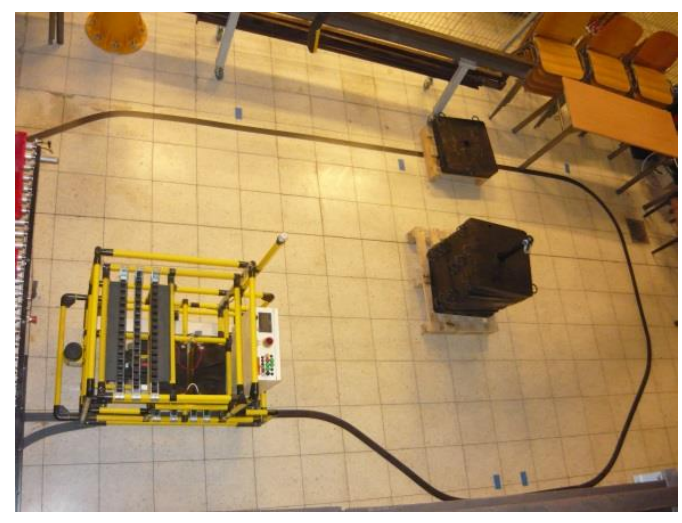

Fig. 5. Aerial view of the AGV system

Table 2. Characteristics of the AGV system

\begin{tabular}{|l|c|}
\hline Maximum mass of the built-in coupling bar frame & $400 \mathrm{~kg}$ \\
\hline Direction of the displacement & Forward \\
\hline Guide system & Magnetic stripe \\
\hline Drive system & $2 \mathrm{WD}$ \\
\hline The minimum radius of rotation & $\mathrm{R} 600 \mathrm{~mm}$ \\
\hline Speed & $3 \mathrm{~m} / \mathrm{min}-50 \mathrm{~m} / \mathrm{min}$ \\
\hline Roadway & Approximately 1 degree \\
\hline Obstacle sensor & - \\
\hline Certificate & CE \\
\hline Battery set & $2 \times 12 \mathrm{~V}-100 \mathrm{Ah}$ \\
\hline
\end{tabular}

In this section, the continuous operation scenario of the AGV system is described. The system consists of two conveyor belts at a distance of 19 meters, the AGV has to move a load of $400 \mathrm{~kg}$ from conveyor 1 to conveyor 2 (1-2 direction), and then return to the starting point without the load (2-1 direction). The cycle is repeated continuously until 
completing 3 hours of work. The AGV in the 1-2 direction delays around 360 seconds by the load weight and in the 2-1 direction it only takes 120 seconds when the process does not need to stop it in the return, if the AGV needs to charge the battery in the return (depending on the scenario proposed by the optimization algorithm to be evaluated), in the 2-1 direction it recovers 48 seconds until the charge point, stops for a time $t c$ and then returns to the starting point in 72 seconds. As can be seen, the AGV operation is dynamic during the process and depends on the system conditions, means, the level of the SoC.

\section{MODEL SOLUTION}

In this section, the initial conditions assumed for the problem simulation are described, then the results of two scenarios are discussed, the first one shows the operation capacity of the AGV during three hours of continuous operation without receiving automatic and opportunity charging, and the second one shows the optimization model solutions (four possible solutions) and the same variables of the first scenario, but with the changes introduced by the automatic and opportunity charging.

In the direction 1-2, the AGV system consumes or dry from the battery $18 \mathrm{~A}$, product of the load weight. However, in the direction 2-1 only consumes $5 \mathrm{~A}$. The system presents the following initial conditions:

1. the battery capacity is $1200 \mathrm{Wh}$,

2. the nominal voltage $V_{\text {nom }}$ is $12 \mathrm{~V}$,

3. internal resistance of the circuit is $0.001 \Omega$,

4. the battery capacity at the beginning of the simulation is $70 \%$ of its capacity,

5. the external temperature is $25^{\circ} \mathrm{C}$,

6. fundamental sample time $T s=0.01$ seconds,

7. capacitance of the circuit $C d c=0.001 \mathrm{~F}$,

8. initial capacitor voltage $V c d c_{0}=0.95 \times V_{\text {nom }}$ in $\mathrm{V}$,

9. simulation window of a work cycle is 3600 seconds (1 hour),

10. the simulated scenario has three working hours, and each hour has nine closed circuits.

\subsection{SCENARIO 1: WITHOUT AUTOMATIC AND OPPORTUNITY CHARGING}

Figure 6 shows the current over the time, as we can see the current is the model input variable and determines the SoC of the battery (model output and indicator that describe the operation capacity of the AGV), in the direction 1-2 the AGV system consumes 18 A and in the direction 2-1 consumes 5 A. Figure 7 shows the SoC behaviour over the time in the battery, and as we can see the consumption is proportional to the current intensity demand by the AGV system.

Figure 8 shows the variable SoC after three hours of continuous work. Visible is the bound red line in the diagram. Therefore, in the scenario 1 the AGV system do not complete the cycle of three working hours planned because the ending point of the blue line is below to the bound red line. Figure 8 is the evidence that the AGV need charge the battery during 
the working cycle to successfully completed three hours of continuous work, and the problem to be solve is find the time $t c$ and the number of charging moments during three hours of continuous work, affecting as little as possible the time to finish the mission.

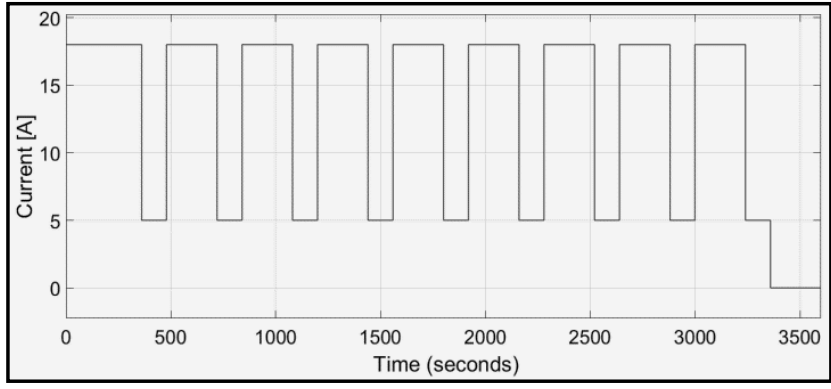

Fig. 6. Current behaviour in scenario 1

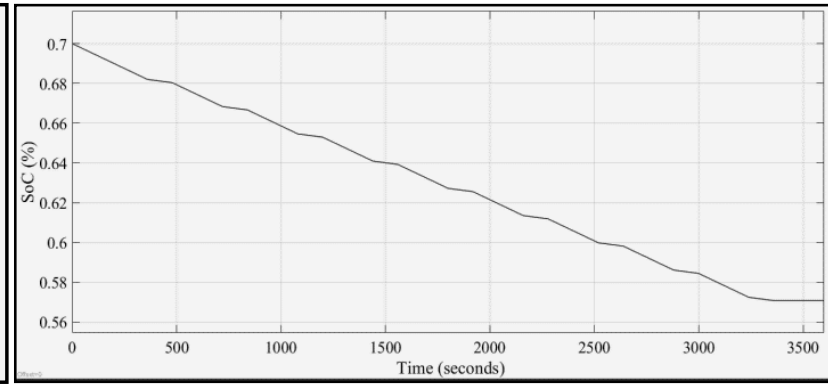

Fig. 7. SoC behaviour in scenario 1

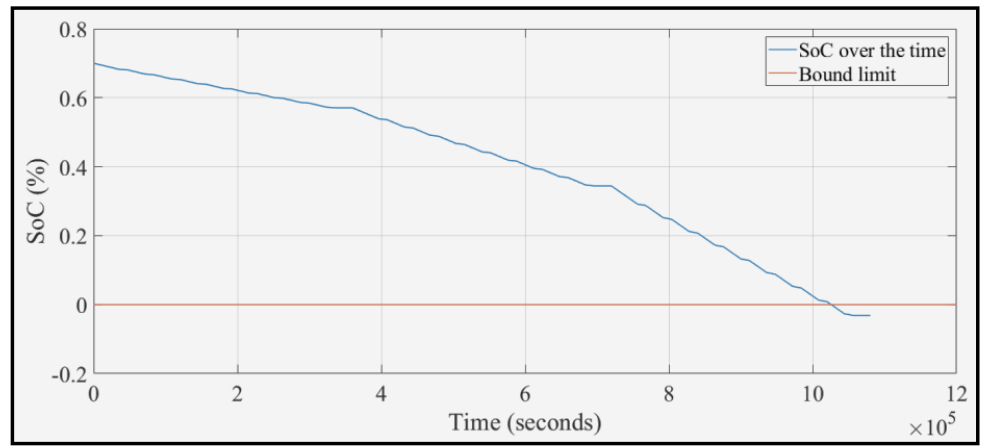

Fig. 8. SoC behaviour of the scenario 1 during three working hours

\subsection{SCENARIO 2: WITH AUTOMATIC AND OPPORTUNITY CHARGING}

In this scenario, it is assumed that the source can deliver power of $1200 \mathrm{~W}$ for a time $t c$ (during the implementation process the $t c_{\max }=100$ seconds) defined by the user. Given the four different optimization objectives described in the section, Table 3 describe the solutions using the targets $6,6 \mathrm{a}, 6 \mathrm{~b}$ and $6 \mathrm{c}$.

Table 3. Scenario solutions

\begin{tabular}{|c|c|c|}
\hline Targets & tc & Ntc \\
\hline 6 & 4 & 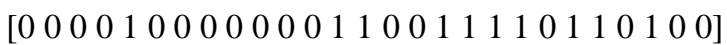 \\
\hline $6 \mathrm{a}$ & 16 & 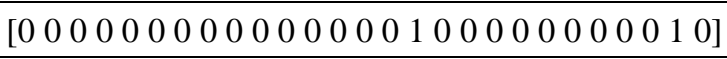 \\
\hline $6 \mathrm{~b}$ & 4 & 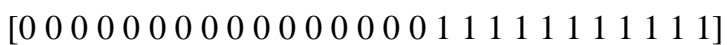 \\
\hline $6 \mathrm{c}$ & 34 & 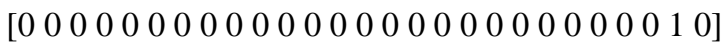 \\
\hline
\end{tabular}

Coherence between comparable targets was noted. Between unweighted targets, the results of the optimization give a set of equivalents stopped charging times during the process (see Table 3 , cases 6 and $6 \mathrm{~b}$ ), but target $6 \mathrm{~b}$ selects the charging time in the last 
few work cycles because the optimized function is area under the curve. However, the number of times results higher than expected. In both cases, the AGV stops several times. The weighted targets result more aligned with expected outcome of the experiments.

The targets $6 \mathrm{a}$ and $6 \mathrm{c}$ are two possible well-behave solutions for this problem. The selection of the best target is subject to analyst's adjustment, and just to shows the outcome of the optimization model, in the following paragraphs we describe all the variables of the circuit using the target $6 \mathrm{c}$.

As a result of the optimization model it was obtained that the AGV system needs one charging moments in the work cycle before the last for only 34 seconds, as describe Fig. 9 convergence and Table 3 target $6 \mathrm{c}$. Figure 10 shows the current behaviour, as we can see the battery receives one moment of charge in the work cycle before the last. Figure 11 shows the $\mathrm{SoC}$ behaviour in the battery. Where we can observe the small increments in the SoC product of the battery charge. Figure 12 shows how it is necessary that the battery receives charge during the proposed scenario. If the battery does not receive a charge during the process, the AGV system does not fulfil the mission. However, the optimization model shows the minimum necessary condition for the AGV system to fulfil the mission, in this case, one charging moments during 34 seconds in the work cycle before the last.

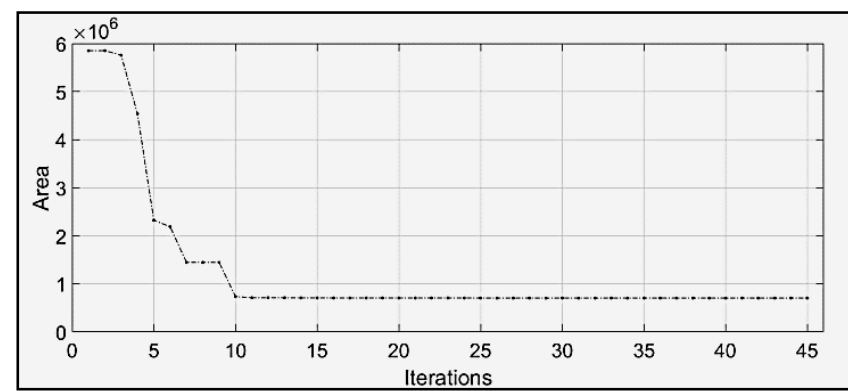

Fig. 9. Convergence of the scenario $6 c$

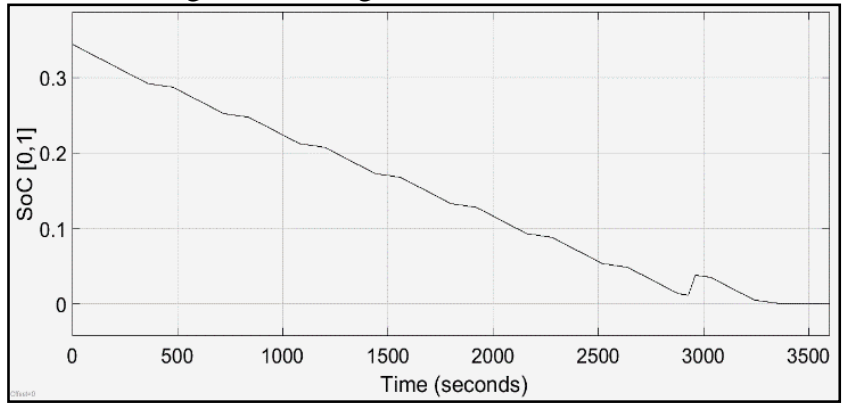

Fig. 11. SoC behaviour in scenario 2

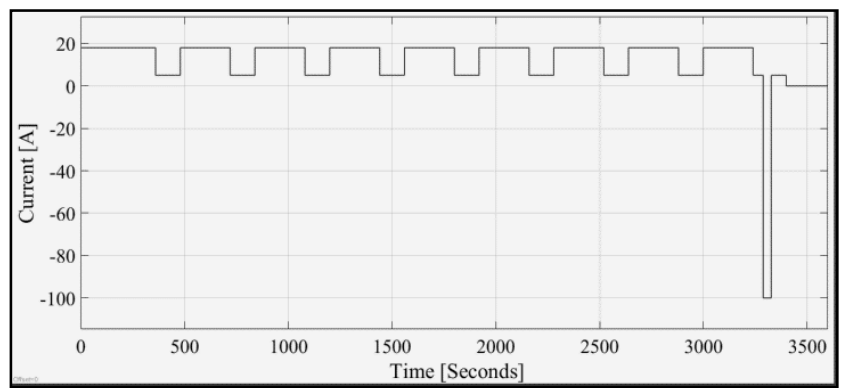

Fig. 10. Current behaviour in scenario 2

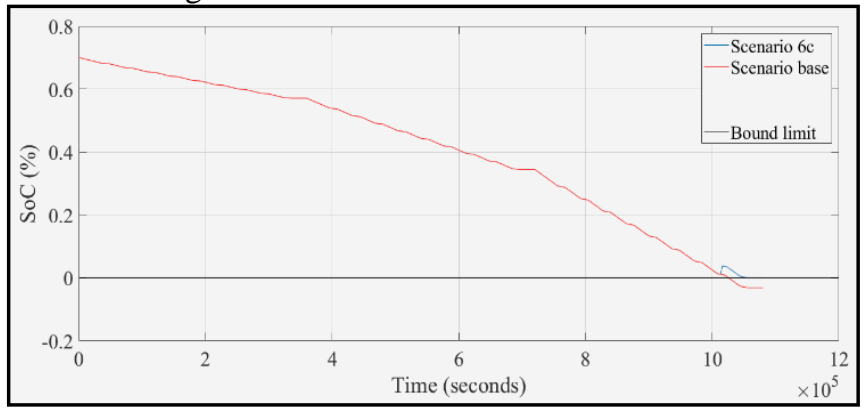

Fig. 12. SoC behaviour in both scenarios during three working hours

\section{CONCLUSION}

The investigation results show the best planning trajectory for an AGV system, energy-oriented approach, to complete three hours of continuous operation under the conditions considered. The selected SoC indicator fully links the planning trajectory or path problem with the energy access needed by the AGV to complete the mission, and more 
when the selected variable is subject of optimization. Between the four criteria evaluated, weighted cases result more accurate in this problem, and the objective $6 \mathrm{c}$ behave as an expected potential real scenario. The proposed optimization model allows estimating the operation capacity of the AGV through the SoC indicator according to the proposed scenario, therefore, it is a useful tool for the analysis of AGV systems. The results support the process of making decisions and create the bases to analyse the real system behaviour. In future research steps, all the simulated results will be tested in a laboratory-scale experimental environment. Also, implementation into intelligent functions development on autonomous electric vehicle platform [17] are planned.

\section{ACKNOWLEDGEMENTS}

The work has been financially supported by the Polish Ministry of Science and Higher Education.

\section{REFERENCES}

[1] SZPYTKO J., 2004, Integrated Decision Making Supporting the Exploitation and Control of Transport Devices. Kraków, Uczelniane Wydawnictwa Naukowo-Dydaktyczne AGH, (in Polisch).

[2] BRUCE G., RAGHAVAN S., WASIL E., 2008, The Vehicle Routing Problem: Latest Advances and New Challenges, Springer.

[3] HUANG Y., LIANG CH., YANG Y., 2009, The Optimum Route Problem by Genetic Algorithm for Loading/Unloading of Yard Crane, Computers \& Industrial Engineering, 56/3, 993-1001.

[4] NISHI T., MORINAKAB S., KONISHIB M., 2007, A Distributed Routing Method for AGVs Under Motion Delay Disturbance, Robotics and Computer-Integrated Manufacturing, 23/5, 517-532.

[5] QIU L., HSU W.J, HUANG S.Y., WANG H., 2002, Scheduling and Routing Algorithms for AGVs: a Survey, Int. J. Prod. Res., 40/3, 745-760.

[6] NEWMAN P.M., DURRANT-WHYTE H.F., 2001, A New Solution to the Simultaneous Localization and Map Building (SLAM) Problem, IEEE Transactions on Robotics and Automation, 17/3, 229-241.

[7] SZPYTKO J., HYLA P., 2011, Automated Guided Vehicles Navigating Problem in Container Terminal, Logistics and Transport, 13/2, 107-116.

[8] HYLA P., SZPYTKO J., 2017, Automated Guided Vehicles - the Survey. Journal of KONES: Powertrain and Transport, 24/3, 101-110,

[9] FARAHANI Z.F., LAPORTE G., MIANDOABCHI E., BINA S., 2008, Designing Efficient Methods for the Tandem AGV Network Design Problem Using Tabu Search and Genetic Algorithm, Int. J. Adv. Manuf. Technol., 36, 996-1009.

[10] SCHMIDT J., MEYER-BARLAG C., EISEL M., KOLBE L.M., APPELRATH H.J., 2015, Using BatteryElectric AGVs in Container Terminals-Assessing the Potential and Optimizing the Economic Viability, Research in Transportation Business \& Management, 17, 99-111.

[11] KABIRA S.Q., SUZUKI Y., 2018, Increasing Manufacturing Flexibility Through Battery Management of Automated Guided Vehicles, Computers \& Industrial Engineering, 117, 225-236.

[12] ZHAN X., XU LI, ZHANG J., LI A., 2019, Study on AGVs Battery Charging Strategy for Improving Utilization, 52nd CIRP Conference on Manufacturing Systems, Procedia CIRP, 81, 558-563.

[13] MA N., ZHOU C., STEPHEN A., 2021, Simulation Model and Performance Evaluation of Battery-Powered AGV Systems in Automated Container Terminals, Simulation Modelling Practice and Theory, 106, $102-146$.

[14] MATLAB, 2010, version 9.7.9.1319299 (R2019b), Natick, Massachusetts: The MathWorks Inc.

[15] KENNEDY J., EBERHART R., 1995, Particle Swarm Optimization, Proceedings of the IEEE International Conference on Neural Networks, Perth, Australia, 1942-1945.

[16] MEZURA-MONTES E., COELLO C.A., 2011, Constraint-Handling in Nature-Inspired Numerical Optimization: Past, Present and Future, Swarm and Evolutionary Computation, 173-194.

[17] WANG R., SELL R., RASSOLKIN A., OTTO T., MALAYJERDI E., 2020, Intelligent Functions Development on Autonomous Electric Vehicle Platform, Journal of Machine Engineering., 20/2, 114-125. 